\title{
Automating Loading and Locking of New Generation Air-cargo Containers
}

\author{
Georgios Bolanakis ${ }^{1, *}$, Konstantinos Machairas ${ }^{1}$, Konstantinos Koutsoukis $^{1}$, Athanasios Mastrogeorgiou ${ }^{1}$, \\ Michael Loupis ${ }^{2,3}$, and Evangelos Papadopoulos ${ }^{1}$ \\ ${ }^{1}$ Control Systems Lab - National Technical University of Athens, 15780 Athens, Greece \\ ${ }^{2}$ Scandinavian Avionics Greece S.A., Athens International Airport, 19019 Spata, Greece \\ ${ }^{3}$ National \& Kapodistrian University of Athens, Evripou Campus, Psachna 34400, Greece
}

\begin{abstract}
In this paper, an outline of NTUA's work in the framework of project INTELLICONT is presented. We describe the current state of the air-cargo handling procedures and how the autonomous system that is under development is going to simplify these and increase the overall efficiency. Important issues and challenges regarding the system's development are discussed and a preliminary design of the novel robotic platform is given. The main tasks of this platform include the autonomous motion and locking of containers with mass exceeding one tone, avoiding at the same time obstacles and surpassing terrain discontinuities. Information regarding the selected actuators and other key electrical components, such as motor drivers and sensors are provided also. The architecture of the embedded system and the specifications of the selected Central Control Unit are described, as well as the integration of the motor drivers, sensors and other peripherals with the Robot Operating System (ROS). Further details on the development of a high accuracy localization system, which is mandatory to lock the container safely to the corresponding positions are provided also. In addition, we give details regarding the locking mechanism with integrated monitoring functionalities, an important part of the system. Simulation experiments validate the selected position controller and key system specifications are highlighted based on results. Finally, recent prototype experiments conducted to verify the localization system are presented.
\end{abstract}

\section{Introduction}

Over the last decades, the air-cargo industry has experienced tremendous growth [1-2]. However, the most essential components, the air-cargo containers or Unit Load Devices (ULDs), have not followed the same evolution as the aircraft structures and systems, and remain open to technological advancements. In the meantime, the introduction of autonomous mobile robots and automated guided vehicles has already simplified and accelerated the procedures in fields related to transportation and logistics, especially in complex environments such as warehouses, distribution centres and even hospitals [3-5].

\subsection{Current Air-Cargo Handling}

Currently, air-cargo handling procedures involve mostly manual operations and require preparations and actions made by qualified personnel in order to safely load and unload the ULDs. Also, a great disadvantage lies on the requirement of having aircraft-embedded hardware in the Cargo Compartment (CC) of the aircraft, including multiple power drive units for moving the ULDs (Figure 1). This increases hardware implementation complexity and adds unnecessary weight to the aircraft. In addition, regular maintenance is needed for ensuring smooth operation and avoiding common issues, such as blocked containers in the CC. Indeed, to safely unload these, often this results in broken parts of the aircraft-embedded hardware.

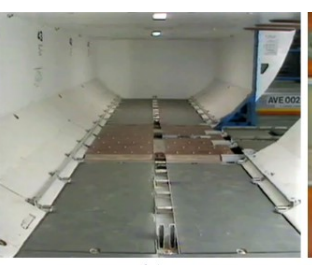

(a)

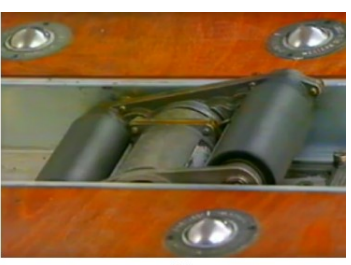

(b)
Fig. 1. (a) Typical Cargo Compartment. (b) Power transfer units inside the A320 CC.

\subsection{Objectives}

With an aim to reduce emissions and weight as well as to increase the overall efficiency, state of the art solutions to the current air-cargo handling procedures are studied. The foremost objective is to simplify and accelerate the loading and unloading procedures. To this end, a semiautonomous robotic system with integrated monitoring capabilities is proposed to eliminate the need for operation by specialized personnel. The new design aims also at reducing the aircraft-embedded hardware to a minimum, to decrease the required maintenance, and to increase efficiency both time-wise and energy-wise.

\footnotetext{
* Corresponding author: gbolanakis@hotmail.com
} 
The initial state of the loading procedure is shown in Figure 2. At first, the Robotic Platform (RP), shown in blue, and the ULD are placed on the Cargo Loader (CL). Then, the RP receives a command from an HMI interface to move the container to a specific locking position. The RP moves closely to the ULD, aligns with it and restrains it with a special mechanism located at its centre. In the final steps, the ULD is transferred accurately and locked to the corresponding locking position. A reversed process is performed to unload the container. The weight of the container is supported by special ball or roller surfaces.

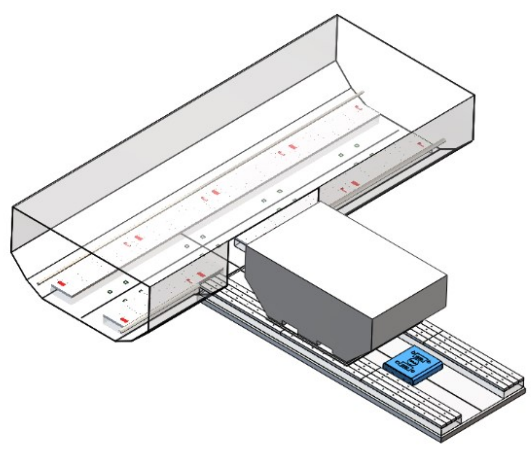

Fig. 2. General concept overview.

\subsection{Challenges and Critical Issues}

Multiple contradictory requirements, geometrical uncertainties and constraints make the design of the particular robotic system a very challenging task. First, determining the final dimensions of the RP is subject to many constraints. For example, the RP height should be minimized in order to maximize the load capacity of the containers. At the same time, enough free space inside the platform must be available to fit high torque motors, a restraint mechanism, batteries and other bulky elements. Additional limitations for the RP dimensions are imposed by the cargo loading procedure, when significant percentage of the ULD baseplate is in the air. More specifically, the ball-mat surfaces that support the ULD at the entrance level must be large enough to guarantee that the ULD stays parallel to the floor at all times, but at the same time allow enough space for the RP to manoeuvre when needed (Figure 3).

Another challenging requirement is that the RP must be able to move ULDs with over 1 tone of mass while surpassing terrain discontinuities such as the gap between the CL and the CC. Moreover, in special situations, e.g. while aligning with the locking position, motions of $1 \mathrm{~mm}$ accuracy need to be performed. To this end, a robust, highly accurate localization system is proposed that uses visual landmarks installed at specific locations.

A matter of great interest, is the design of a locking mechanism that ensures equal force distribution to the aircraft's chassis, guarantees restraint on all axes, and minimizes the mass of the aircraft-embedded hardware interfaces. While trying to overcome the abovementioned challenges, the robotic system shall also maintain backwards compatibility with older aircrafts, a fact that reduces the available design options significantly.

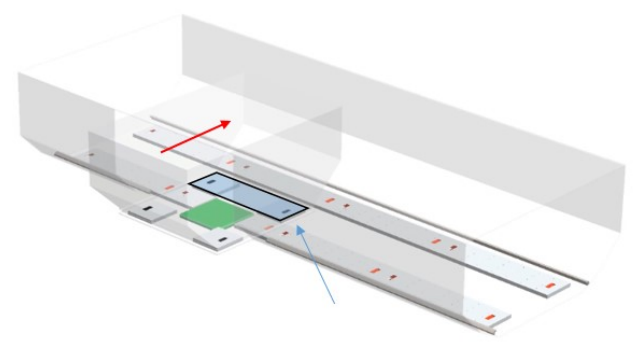

Fig. 3. At the entrance, significant percentage of the ULD baseplate is in the air. The arrow shows a gap which the system must overcome without tumbling.

\section{System Design}

In this section, an outline of the most important specifications of the system is presented. An overview of the current electromechanical design of the RP is given, along with details for the locking mechanism. It is noted that several features of the RP and its sub-systems will be subject to changes, since this is a preliminary design; the final system specifications will be determined based on the results of future experiments.

\subsection{Determining System Specifications}

As a first step in the design process, the most critical system requirements were analysed. For instance, the RP shall be able to move ULDs weighing up to 1.2 tons of mass, identify them using visual aids, perform precise motions across $\mathrm{x}$ and $\mathrm{y}$ axes with longitudinal slope up to $\pm 1.55^{\circ}$, monitor and transmit useful information. Regarding the locking system, it is required to restrain the ULDs along all axes, and in case of power loss or other system malfunction, keep them restrained, providing manual release to the user.

Having specified the key requirements for the robotic system, alternate locomotion concepts were studied including various types of wheels (mecanum, omniwheel etc.), and other electromechanical elements. Among those, a solution that optimally fits the application purposes is presented in detail in the following sections. Simulation experiments are conducted to validate the selected architecture, to test worst case scenarios and to reveal possible weaknesses.

As an example, an open-loop simulation experiment was conducted to define the maximum torque per driving axis needed for the RP to move the ULD across the gap between the $\mathrm{CL}$ and the $\mathrm{CC}$ (Figures 4 and 5). A horizontal gap of $2 \mathrm{~cm}$ and a vertical gap of $0.5 \mathrm{~cm}$ were used as reasonable values that can be observed in existing systems. The particular experiment helped to designate key characteristics of the RP, and also revealed that 
occasionally some wheels of the RP may not touch the ground. Based on the results, the initial specifications were reconsidered including the required mass and number of actuated wheels of the RP.

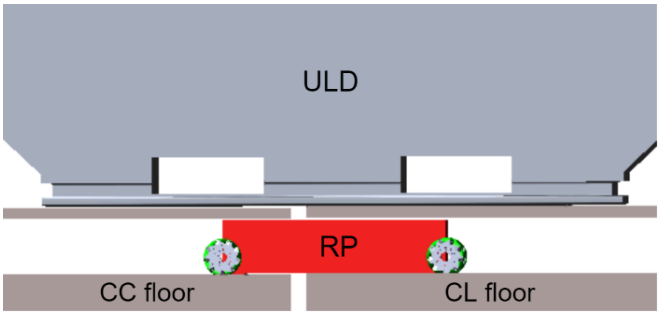

Fig. 4. ADAMS simulation experiment. Motion to the left.

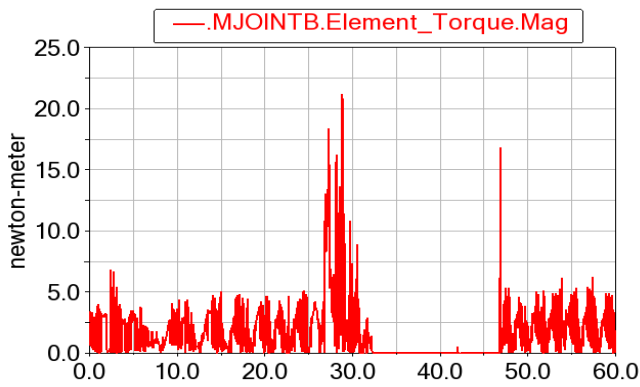

Fig. 5. Motor torque, front wheels not touching the ground.

\subsection{Mechanical/ Electrical Subsystem}

A preliminary design of the RP is shown in Figure 6 . The $\mathrm{RP}$ is designed to fit in a $600 \times 580 \mathrm{~mm}$ envelope in order to move with ease at the CC empty space underneath the ULDs. As far as the RP height is concerned, the distance between the top RP surface and the ground is selected equal to $150 \mathrm{~mm}$ and the chassis height equal to $144 \mathrm{~mm}$. Moreover, its mass exceeds $150 \mathrm{~kg}$ by design; this is required to prevent the RP from slipping while moving the containers on sloped surfaces.
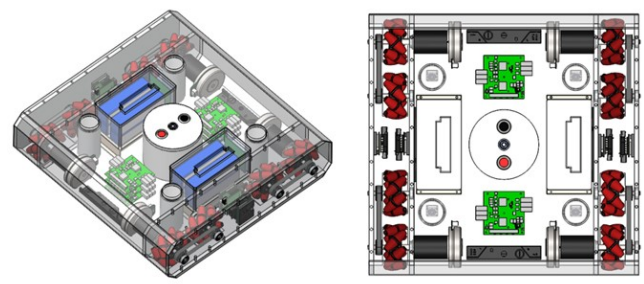

Fig. 6. Preliminary design of the Robotic Platform.

The current platform design employs eight mecanum wheels actuated in pairs by four motors. This type of wheels was selected over other architectures, including omniwheels and swerve drives, as they provide high load capacities in a compact design and are simpler to control [6]. A suitable Maxon actuator is selected such that it features adequate speed-torque capabilities. Particularly, the candidate actuator is comprised of an EC 90 flat brushless motor and a Maxon GP52C planetary gearhead with ratio 53:1, driven by the SBL2360 motor controller by RoboteQ. Each controller integrates high-current power drivers for two brushless DC motors at up to $30 \mathrm{~A}$ output current per channel.

A restraint mechanism for the containers is located at the centre of the RP. Using this mechanism, the RP does not lift the ULD mass but only drags it along the desired paths by exerting on it forces parallel to the floor. To this end, two cylindrical appendages emerge from the RP top surface and are inserted into two slots (of slightly larger diameter) at the ULD baseplate. This mechanism will be actuated using a brushless DC motor paired with a ballscrew transmission mechanism.

Valuable sensory feedback will be provided to the control system by four Magnetic Guide Sensors (MGS), two for sensing lateral distance across the $\mathrm{x}$ axis and two for $y$ axis. For critical localization tasks eight image sensors, four directed upwards and four downwards, are placed at the corners of the RP. An Intel Barebone NUC8i7HNK will be used as the Central Control Unit of the RP. Among other features, this includes an i7-8705G $8^{\text {th }}$ Generation CPU, 16GB DDR4 RAM, an integrated graphics card and up to 13 USB ports.

Regarding the power and charging system, the RP will be powered by two battery modules (Figure 7) allowing an average continuous operation of 150 minutes. The battery modules are designed to be easily removable and replaceable by supervisory personnel. Each of them consists of high energy density LiPO cells, providing exceptional charge/ discharge current capacities and great lifespan (over 500 charge cycles). An integrated Battery Management System (BMS) comes along with the battery module, transmitting useful sensory data to the system and features cell balancing functions. Custom fastcharging stations, installed on convenient locations outside the aircraft, will be able to re-energize a battery module within 60 minutes.

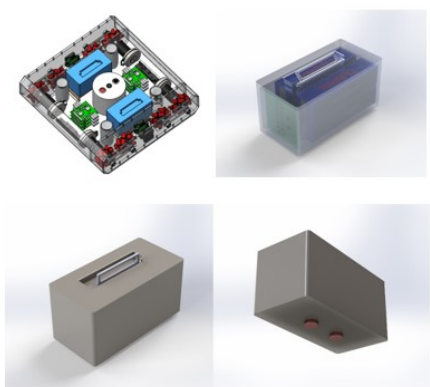

Fig. 7. Removable Battery Modules with integrated Battery Management System.

\subsection{Embedded System Architecture}

The embedded system of the RP ties together the different subsystems and takes over complex tasks, the most important being the accurate motion inside and outside the $\mathrm{A} / \mathrm{C}$ using feedback provided by the installed sensors. An 
overview of the embedded system is given in Figure 8 . The Central Control Unit (CCU) is composed by an Intel Barebone PC running Robot Operating System (ROS) on Ubuntu Linux [7].

Hardware components by RoboteQ, including the MGSs, the motor controllers and the BMS are attached to a RoboCAN network, a RoboteQ's proprietary meshed networking scheme allowing multiple devices to operate together as a single system. By connecting to any device through RS232, the CCU has access to any device connected to the RoboCAN network. Eight image sensors are connected via USB to the CCU. Some share the same USB bus without decreasing bandwidth performance.

Separate ROS nodes have been developed for interfacing with each image sensor, publishing to the corresponding topics at $50 \mathrm{~Hz}$ rate. A special node is being used for communicating with the devices of the RoboCAN network in order to receive feedback from the MGSs and send commands to the motor controllers.

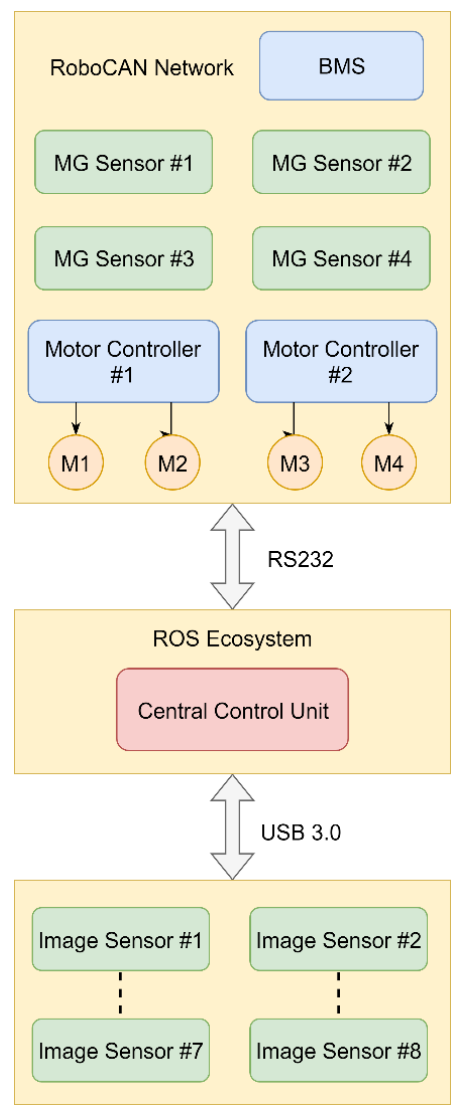

Fig. 8. Embedded system architecture overview.

\subsection{Locking Mechanism and Counterparts}

The locking mechanism is an important part of the system, since it provides automated restraining capabilities to the containers. The mechanism involves the development of two separate sub-systems, as shown in Figure 9. The first one is completely passive and is installed on the floor of the CC (counterparts). The other sub-system is installed on the baseplate of the ULD, and may be activated, whenever needed, over the restraint mechanism of the RP.

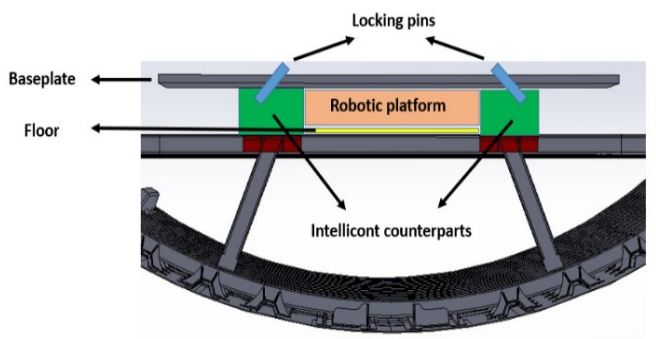

Fig. 9. Locking Mechanism Concept. Only the baseplate of the container is shown in this figure; the rest is omitted.

More specifically, the locking mechanism consists of electrical actuators, for changing the states of the four locking pins, along with sensors of different types to ensure accurate reading of the locking state (Figure 10). The locking pins are located at the four corners of the ULD and the $45^{\circ}$ design ensures restrain on all axes. In case of system malfunction, a manual release will be available to be used by the supervisory personnel. Two Hall-effect sensors and two mechanical end-stops are installed in every corner of the baseplate and permanent magnets are placed inside the locking pins for changing the state of the sensors accordingly. This design ensures a robust monitoring system and allows to detect situations where pins are blocked at an intermediate location.

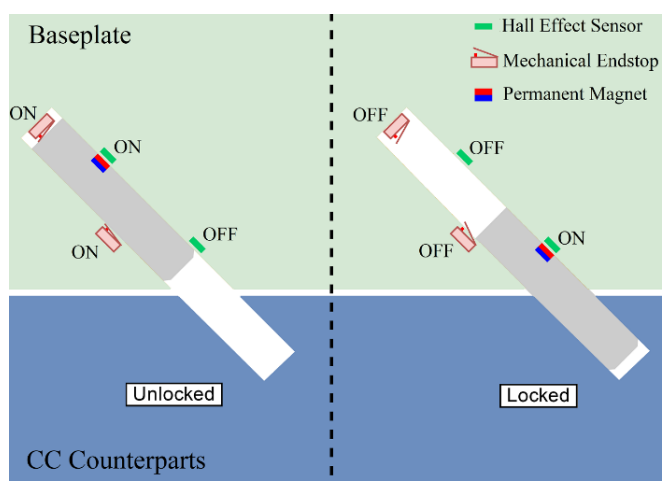

Fig. 10. Locking mechanism state monitoring based on Halleffect sensors and mechanical endstops.

\section{Motion Control}

The autonomous motion of the RP is divided into two main modes of operation. First, at some stages, high speed navigation is needed. At these stages, the line following technique is used with feedback given by the MGSs that detect the predefined magnetic tracks installed on the floor of the CL and the CC. 
In critical localization tasks, the real time localization system based on the image sensors is employed. This system is used primarily in two situations, during which accuracy is the highest priority: when the RP aligns with and under the container, and just before activating the locking mechanism, while aligning with locking position.

\subsection{Sensory System}

Sensing is essential for the RP. To safely move and lock the container, the RP shall know precisely its position at all times. The localization system is physically implemented using two types of sensors. Feedback given by magnetic guide sensors is used to easily navigate at high speeds inside the CC as well as on CL. Magnetic sensors are widely used in the development of automated guided vehicles [8-9]. The RoboteQ MGSs (Figure 11) give the lateral distance of the platform with respect to the center of the magnetic line with a resolution of $1 \mathrm{~mm}$ at $100 \mathrm{~Hz}$ update rate.

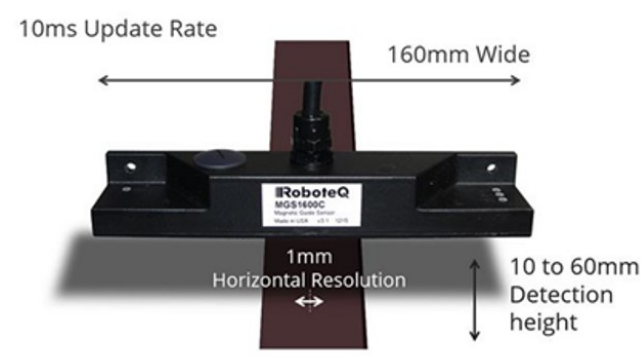

Fig. 11. Magnetic Guide Sensors by RoboteQ, used for line following based navigation.

Additionally, they provide lateral sensing area of 16 $\mathrm{cm}$ and they are able to detect magnetic markers installed next to the magnetic line, allowing the RP to slow down when reaching critical locations. These sensors were selected mainly because they are immune to dirt and light invariances. Other technologies, such as Infra-red Ray (IR), would fail in the conditions of the studied application

A mock-up of the RP was built, which will be presented in detail in the next chapter. The mock-up RP is equipped with a Sony PlayStation Eye Camera which provides a refresh rate of up to $60 \mathrm{~Hz}$ through USB 2.0 interface (Figure 12).

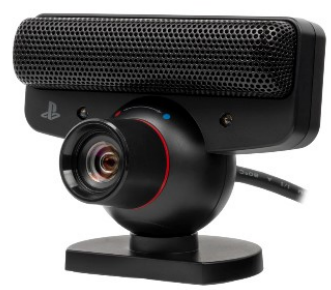

Fig. 12. Sony PlayStation Eye Camera used for validating the localization system on the prototype robotic platform.
However, in the final design, USB 3.0 image sensors will be employed instead, to avoid bandwidth performance issues when multiple sensors are operated simultaneously. Custom modifications are made to the lens of the camera for adjusting the focal point accordingly. It is expected that the localization system will be able to provide position feedback $(\mathrm{x}, \mathrm{y}, \theta)$ at $1 \mathrm{~mm}$ and $0.2^{\circ}$ respectively.

\subsection{Localization System}

The localization system currently developed is based on QR Code Labels, the most widely used two-dimensional barcodes. QR Code labels have been used in the past to assist robot navigation in indoor environments [10-13]. In the studied application, a prerequisite for the system operation, is the installation of permanent QR-code grids at ULD baseplates and CC locking positions that will be used as visual landmarks for the image sensors. Each QR code will provide information regarding its position on a cartesian system defined by the grid (Figure 13).

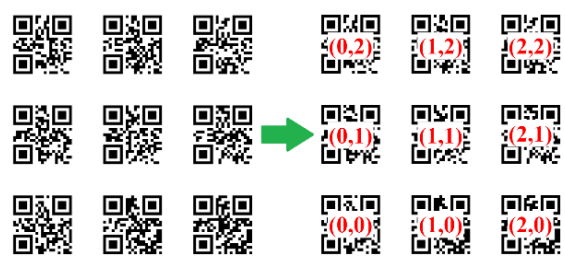

Fig. 13. QR code grid.

At least one image sensor with predefined offsets from the centre of the RP is needed to implement this technique. By recognizing one or more $\mathrm{QR}$ code labels, the RP can determine its $\mathrm{x}, \mathrm{y}$ and $\theta$ offsets with respect to the coordinate system of the container (Figure 14). The main advantage of this method is that it provides feedback at a high frequency rate allowing the use of a position controller.

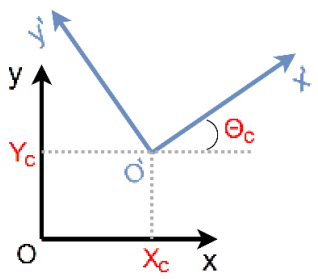

Fig. 14. The Cartesian coordinate systems $x y$ and $x^{\prime} y^{\prime}$ of the container and the RP respectively.

\section{Simulation Experiments}

Simulation experiments were performed in MATLAB Simulink in order to evaluate different types of controllers for the RP and reveal possible weaknesses of the current system design. The foremost requirements of the experiments were to maintain system's stability while achieving minimum steady state error, so that the container can be locked safely. 


\subsection{Model}

A simple model of the system was developed, consisting of the RP moving the ULD across the ball transfer units (Figure 15). Motion only across the $x$ axis is considered and it is assumed that the mass of the RP and the friction coefficient of the floor are such that prevent the RP from slipping. Disturbances such as white noise, latency, and coulomb and viscous friction are included in the model.

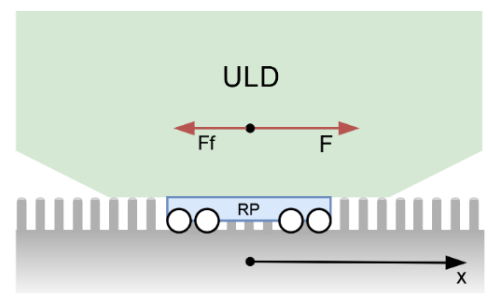

Fig. 15. Simple model of the RP moving the ULD across the ball transfer units.

\subsection{Controller Selection}

Selecting the appropriate controller can be frustrating for systems like this that include non-linear disturbances like friction. Initially a PID controller was used, but transient response of the system showed undesirable effects due to the proportional and derivative kick. To address this issue, a modified version of the PID controller, known as the IPD controller (Figure 16), was finally selected [14].

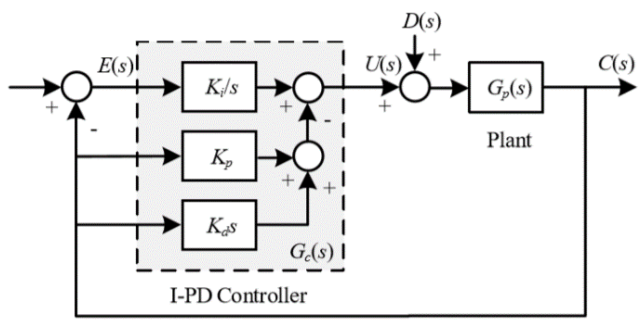

Fig. 16. Primitive control loop with an I-PD controller.

\subsection{Simulation Results}

Considering the duration of the motion ( $10 \mathrm{~s})$, the mass of the containers (1.2 tons), and the coefficient of friction between the floor and the wheels $\left(\mu_{\mathrm{f}}=0.8\right)$ as constants, experiments were conducted varying the coefficient of friction between the container and the ball transfer units $\left(\mu_{b}\right)$, the slope $(\varphi)$, and the target point $\left(X_{t}\right)$ of the trajectory feeding the controller. The results are shown in Table 1.

In the worst-case scenario $\left(\varphi=1.55^{\circ}, \mathrm{X}_{\mathrm{t}}=0.3 \mathrm{~m}, \mu_{\mathrm{b}}=\right.$ 0.08 ), the maximum output force of the controller was $1523 \mathrm{~N}$, and the minimum RP mass required to avoid slipping was calculated to be $194.4 \mathrm{~kg}$. The desired and the actual ULD position regarding this case are shown in
Figure 17. Also, the force output of the I-PD controller, and the system velocity are shown in Figures 18 and 19 respectively.

Table 1. Simulation results.

\begin{tabular}{|r|r|r|r|r|}
\hline \multicolumn{1}{|c|}{$\boldsymbol{\varphi}\left(\mathbf{(}^{\mathbf{})}\right.$} & $\mathbf{X t}(\mathbf{m})$ & \multicolumn{1}{c|}{$\boldsymbol{\mu}_{\mathbf{b}}$} & $\mathbf{F}_{\mathbf{m a x}} \mathbf{( N )}$ & $\begin{array}{c}\text { m required } \\
(\mathbf{k g})\end{array}$ \\
\hline 0 & 0.1 & 0.01 & 205 & 26.1 \\
\hline 0 & 0.1 & 0.05 & 704 & 89.8 \\
\hline 0 & 0.1 & 0.08 & 1074 & 137.0 \\
\hline 0 & 0.3 & 0.01 & 239 & 30.5 \\
\hline 0 & 0.3 & 0.05 & 739 & 94.3 \\
\hline 0 & 0.3 & 0.08 & 1104 & 140.9 \\
\hline 1.55 & 0.1 & 0.01 & 546 & 69.7 \\
\hline 1.55 & 0.1 & 0.05 & 1075 & 137.1 \\
\hline 1.55 & 0.1 & 0.08 & 1478 & 188.5 \\
\hline 1.55 & 0.3 & 0.01 & 578 & 73.8 \\
\hline 1.55 & 0.3 & 0.05 & 1107 & 141.2 \\
\hline 1.55 & 0.3 & 0.08 & 1523 & 194.4 \\
\hline-1.55 & 0.1 & 0.01 & 497 & 63.4 \\
\hline-1.55 & 0.1 & 0.05 & 867 & 110.7 \\
\hline-1.55 & 0.1 & 0.08 & 1146 & 146.2 \\
\hline-1.55 & 0.3 & 0.01 & 498 & 63.6 \\
\hline-1.55 & 0.3 & 0.05 & 857 & 109.4 \\
\hline-1.55 & 0.3 & 0.08 & 1126 & 143.6 \\
\hline
\end{tabular}

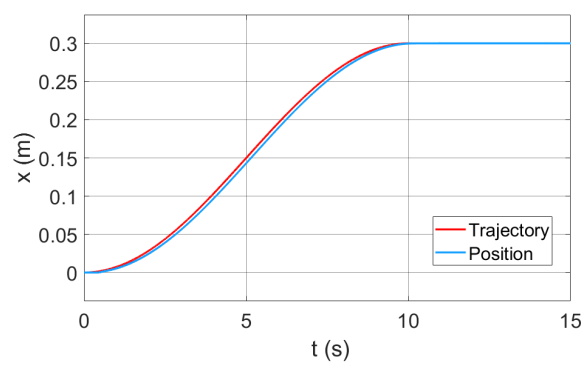

Fig. 17. Desired and actual system trajectory.

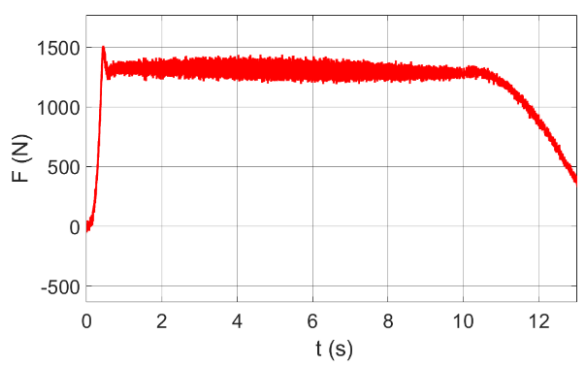

Fig. 18. Force output from the I-PD controller.

The controller's performance in terms of transient response and steady state error $(100 \mu \mathrm{m})$ was considered satisfactory. In particular, as shown in Figure 17, the given trajectory is precisely followed by the RP and the motion is performed in an average speed of $3 \mathrm{~cm} / \mathrm{s}$, while the highest speed reaches the value of $5 \mathrm{~cm} / \mathrm{s}$. The results raise questions with respect to the mass of the RP and the 
maximum required torque that should be available by the actuators. These will be taken into account in the final designs of the robotic system.

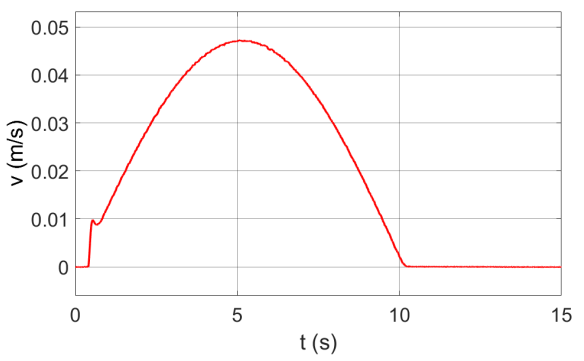

Fig. 19. Velocity of the RP and ULD system.

\section{Hardware Experiments}

\subsection{Robotic Platform Overview}

A prototype robotic platform was developed for validating the selected architecture and testing the localization system throughout its development. The body frame is constructed of aluminum profiles and four mecanum wheels, able to support the mass of up to 180 $\mathrm{kg}$ in total have been symmetrically placed at the corners. In the current version of the prototype, four Maxon DC motors paired with GT2 timing belts are used, providing $8 \mathrm{Nm}$ of continuous torque per wheel. Two SDC2160 motor controllers and two MGS1600GY magnetic guide sensors are attached to the RoboCAN network. A Sony PlayStation Eye Camera, which is located at its center and directed downwards, provides image frames at $50 \mathrm{~Hz}$, while general-purpose LEDs placed at the perimeter, allow the camera to work at max frames per second while minimizing blur effect. Control commands are calculated in a desktop PC and given to the RoboCAN network through an RS232 interface. The system is powered by a bench power supply.

\subsection{Control Algorithm}

In the experiments described next, a simple control algorithm has been used, which provides translational and rotational control of the RP [15]. In every control loop the velocity multipliers for each motor, given by equations (14 ), are calculated. The position controller that is currently used is based on a simple Proportional Controller with proper saturation limits defined, but an IPV controller will be used in future experiments. Three commands are given as inputs to the controller, $\theta_{\mathrm{d}}$ defines the direction at which the RP will move (Figure 20), while $\mathrm{V}_{\mathrm{d}}$ specifies the speed. Lastly, $\mathrm{V}_{\theta}$ sets the rotational speed of the robot.

$$
\begin{aligned}
& V_{1}=V_{d} \sin \left(\theta_{d}+\frac{\pi}{4}\right)+V_{\theta} \\
& V_{2}=V_{d} \cos \left(\theta_{d}+\frac{\pi}{4}\right)-V_{\theta}
\end{aligned}
$$

$$
\begin{aligned}
& V_{3}=V_{d} \cos \left(\theta_{d}+\frac{\pi}{4}\right)+V_{\theta} \\
& V_{4}=V_{d} \sin \left(\theta_{d}+\frac{\pi}{4}\right)-V_{\theta}
\end{aligned}
$$

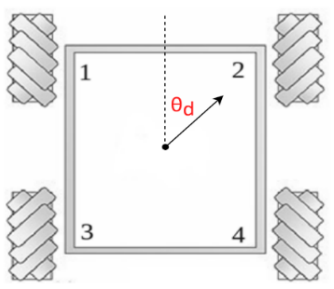

Fig. 20. Direction of the RP motion as given by the selected controller [15].

\subsection{Line Following and directional angle tracking}

Motion control using line following has been validated in preliminary experiments, where the pose of the RP is calculated based on the readings given by the magnetic guide sensors. The position controller targets to minimize the lateral distance $\mathrm{d}$ and the directional angle $\theta$ of the robot with respect to the magnetic line (Figure 21).

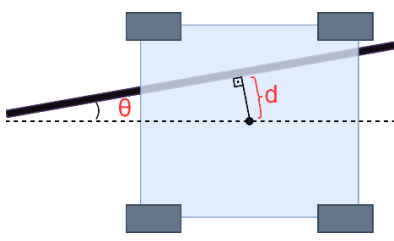

Fig. 21. The errors in lateral distance $\mathrm{d}$ and directional angle $\theta$ while following a magnetic line.

A video with the RP in action while implementing the line following technique may be viewed online [16]. More specifically, in this experiment the RP follows the magnetic line and slows down when it detects a magnetic marker, see Figure 22.

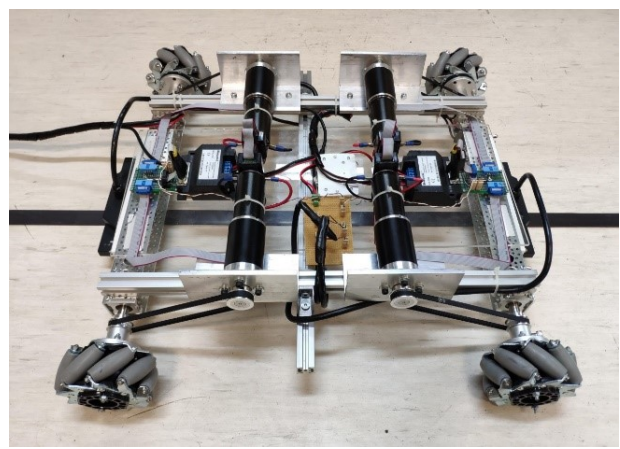

Fig. 22. The prototype RP following the magnetic line.

In addition, experiments for validating the high accuracy localization system based on QR Code Labels have been conducted. These experiments focus on tracking the directional angle of the RP with respect to the coordinate system defined by a QR Code grid which is located beneath it, Figure 23 . 


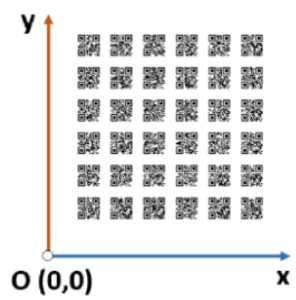

Fig. 23. QR Code grid located underneath the RP.

In this test case, the RP is initially located over the QR Code grid, at a random pose $\left(\theta=18^{\circ}\right)$. A unit step is given as input to the position controller, commanding the RP to eliminate the directional angle offset $\left(\theta=0^{\circ}\right)$. The result given by the localization system is presented in Figure 24 . In future experiments, control on all offsets $(x, y, \theta)$ will be attempted.

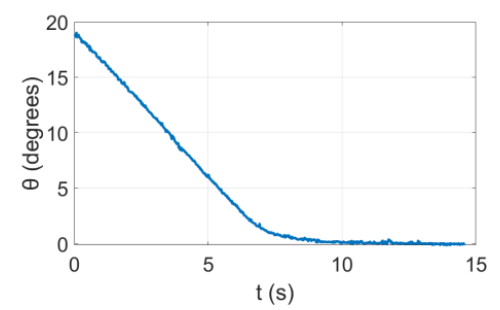

Fig. 24. RP directional angle response in unit step input.

\section{Conclusions}

From the experience gained so far, overall, INTELLICONT is a demanding project; multiple contradictory requirements, geometrical uncertainties and constraints shorten the design options and complicate the determination of the main system characteristics including, among others, the RP's dimensions and mass, the number of actuated wheels and the required motor torque. Air-cargo containers with more than one ton of mass require a high accuracy localization system and advanced control algorithms to be transferred and locked safely to the corresponding parking positions. On the other hand, state-of-the-art technologies such as high accuracy magnetic guide sensors and open source software facilitate our effort. In this work, the key design challenges towards an autonomous air cargo handling robotic system were highlighted, and the initial design choices were described in detail. Simulation experiments were performed aiding in determining key elements of the system. Finally, initial hardware experiments were carried as a first validation of the design choices.

\section{Acknowledgement}

The research work presented in this paper has received funding from the European Union's H2020-EU.3.4.5.1.IADP Large Passenger Aircraft, Research and Innovation Programme, under grant agreement No 785472.

\section{References}

1. "2018 in review - air freight volumes", International Air Transport Association: www.iata.org/economics

2. F. Kiso, A. Deljanin, "Air Freight and Logistics Services.” Pr.-Tr. \& Trans. 21, (2009).

3. K. Azadeh and R. Koster, D. Roy, "Robotized and Automated Warehouse Systems: Review and Recent Developments", Transp. SCI 53, 4 (2017).

4. Y. Wu, D. Ge, "Key Technologies of Warehousing Robot for Intelligent logistics", ISMSS, (2019).

5. W. Wang, C. Du, Z. Du, “A medical transportation robot for carrying and positioning patients between different devices”, Ind. Rob. 46, 6 (2019).

6. F. Adascalitei, I. Doroftei, "Practical applications for mobile robots based on Mecanum wheels - a systematic survey", Romanian Review Precision Mechanics, Optics and Mechatronics, 21-29 (2011).

7. M. Quigley et. al, "ROS: an open-source Robot Operating System.”, ICRA Workshop on Open Source Software 3 (2009).

8. B. B. Abu Bakar, S. S. Mohmad and I. Adam, "Navigation of an Automated Guided Vehicle Based on Sugeno Inference Engine.", J. of App. Sci. 16, 570579 (2016).

9. S. Kamewaka, S. Uemura, "A magnetic guidance method for automated guided vehicles", IEEE Trans. on Mag. 23, 2416-2418 (1987).

10. H. Zhang et. al, "Localization and navigation using QR code for mobile robot in indoor environment", ROBIO, 2501-2506 (2015).

11. L. Cavanini, "A QR-code localization system for mobile robots: Application to smart wheelchairs", ECMR 2017, 1-6 (2017).

12. S. Lee et. al, "QR-code based Localization for Indoor Mobile Robot with validation using a 3D optical tracking instrument", IEEE AIM, Busan, 965-970 (2015).

13. S. Babu, S. Markose, "IoT Enabled Robots with QR Code Based Localization”, ICETIETR, (2018).

14. D. Puangdownreong, A. Nawikavatan, C. Thammarat, "Optimal Design of I-PD Controller for DC Motor Speed Control System by Cuckoo Search.”, Proc. Comp. Sc. 86, 83-86 (2016).

15. I. McInerney, "Simplistic Control of Mecanum Drive”, FRC Team 2022 (Il Mathematics and Science Academy).

16. Video to the line following experiment: https://youtu.be/DG3yyTuZ-Xg 